\title{
Editorial: 50 Years of Physical Review A, B, C, and D
}

Back in February, the world was a different place as I drafted an editorial to celebrate the 50th anniversary of four of our great journals: Physical Review A (PRA), Physical Review B (PRB), Physical Review C (PRC), and Physical Review D (PRD). My intent was to focus on thanking our readers, authors, and referees for their dedicated contributions over many years. I hoped to do the same, in person, at meetings throughout the year. But the world changed due to COVID-19, and I am writing this article in a very different frame of mind. I still want to use this opportunity to celebrate our journals' anniversary, and to thank all who contributed to getting us here.

The history of the journals and how they came about is described in articles in APS News [1] and in Physics Magazine [2]. Approximately every two weeks, each journal will continue to highlight landmark papers from their illustrious history and these "Milestones" are being archived on a collection page (for example journals.aps.org/pra/50th).

I encourage everyone to keep up with these highlights throughout the year. I would like to point out some of the papers that led to Nobel prizes, such as: Wilson's development of the theory of critical phenomena in connection with phase transitions (Nobel Prize 1982) and the discovery of giant magnetoresistance (2007), both published in PRB [3,4]; contributions to the ground-breaking experimental methods that enable measuring and manipulation of individual quantum systems (2012) in PRA [5]; and the observation of neutrino oscillations by the SNO collaboration (2015) covered in $P R C$ [6].

In addition, Physics Magazine is publishing one special feature article for each journal, illuminating the original ideas behind the research of three seminal papers and how they impact present-day early career scientists. The features for PRA [7], PRD [8], and PRB [9] have already been published; if you do not already receive the weekly email alerts from Physics Magazine, please sign up at https://physics.aps.org to make sure you do not miss the upcoming special feature for $P R C$.

But while it is great to be able to reflect on a tremendously successful history, we must also to look towards the future. We live in very uncertain times and presently nobody can predict what long-term impact COVID19 will have on the scientific enterprise in general and basic science in particular.

However, one aspect of scientific research dissemination will remain critically important: quality control. This need has been amplified by the current situation as preliminary and unconfirmed results are being widely distributed and causing sometimes dangerous overreactions. While the arXiv posted a specific reminder with respect to COVID-19 related preprints, this warning is more generally applicable: "e-prints posted on arXiv are not peer-reviewed by arXiv; ... . should not be reported in news media as established information without consulting multiple experts in the field" [10].

As the pandemic unfolds all Physical Review journals will continue to offer our readers and authors highquality peer review of the submitted manuscripts to filter and curate the scientific results. As technologies and digital communication evolve, we will stay nimble and flexible as we adjust to the needs and wishes of the community for scientific quality control. 
In summary, let me assure you that the editors and journal staff continue to be committed to professional peer review and editorial manuscript processing in order to fulfill the APS mission: "To advance and diffuse the knowledge of physics for the benefit of humanity, promote physics, and serve the broader physics community."

\section{Michael Thoennessen \\ Editor in Chief \\ American Physical Society}

(2) Published 11 June 2020

DOI: 10.1103/PhysRevB.101.210001

[1] M. Salter and M. Thoennessen, The Back Page: 50 Years of Physical Review A, B, C, and D, APS News 29(2) (2020).

[2] E. K. Carlson, The Physical Review's Split, 50 Years Later, Physics 13, 27 (2020).

[3] K. G. Wilson, Phys. Rev. B 4, 3174 (1971); 4, 3184 (1971).

[4] G. Binasch, P. Grünberg, F. Saurenbach, and W. Zinn, Phys. Rev. B 39, 4828(R) (1989).

[5] D. J. Wineland and W. M. Itano, Phys. Rev. A 20, 1521 (1979).

[6] B. Aharmim et al. (SNO Collaboration), Phys. Rev. C 72, 055502 (2005); 88, 025501 (2013).

[7] J. Thomas, 50 Years of Physical Review A: The Legacy of Three Classics, Physics 13, 24 (2020).

[8] E. K. Carlson, 50 Years of Physical Review D: Making Ripples in Fields and Spacetime, Physics 13, 64 (2020).

[9] J. Thomas, 50 Years of Physical Review B: Solid Hits in Condensed Matter Research, Physics 13, 29 (2020).

[10] https://arxiv.org 